\title{
DESDE LAS REGIONES TAMBIEN SE PUEDE Experiencias de la VIII Región en materias de juventud y municipalidades
}

\author{
MILTON HENRÍQUEZ SIMPSON*
}

\section{COMENTARIO OBLIGADO Y ACLARATORIO}

EL PRESENTE ARTÍCULO PRETENDE - con los ripios propios de este tipo de intentonas - dar formalidad escrita a cierto discurso que hemos desarrollado en nuestra región respecto de lo que debiese ser la génesis y gestión de una política de juventud.

En primer lugar, hablaremos en «nosotros» por cuanto las ideas expuestas a continuación son el fruto de varios años de relaciones laborales, políticas, amicales y de otras índoles que no viene al caso contar, entre el que suscribe y las personas encargadas de coordinar y, muchas veces, inventar con ingenio y cero recursos las actividades que sus municipios (léase alcaldes, concejales y demases) estiman deben ser ofertadas a los jóvenes de cada comuna.

Este «nosotros» está compuesto, principalmente, por los encargados y encargadas de Casas y Oficinas de Juventud Municipales existentes en la VIII Región, por algunos amigos del área juventud de FOSIS, otros amigos concejales y consejeros regionales, de ONGS.

El afán de nuestra exposición no es otro que demostrar que los compromisos bien asumidos y la creatividad siempre pueden vencer

Concejal de Concepción. Presidente de la Comisión de Juventud de la Asociación Chilena de Municipalidades, Capítulo VIII Región. 
barreras administrativas, presupuestos bajos y, lo más importante, el centralismo brutal que caracteriza nuestra gestión de Estado.

Por último indicamos que no abundaremos en nuestra percepción de la realidad juvenil, ni la problemática que enfrenta el gobierno y otros mundos frente al tema, puesto que, participando del excelente seminario organizado por el Departamento Comunal de Jóvenes de Concepción que motiva estas líneas, creemos compartir en gran medida lo expuesto.

\section{NUESTRA EXPERIENCIA}

En la VIII Región la crisis institucional del INJ, a diferencia de otras regiones, no significó la debacle y desaparición de las Casas y Oficinas de la Juventud. Lo anterior, fruto del diseño que aplicamos particularmente en nuestra zona.

Básicamente, este modelo fue exitoso porque, a pesar del carácter de «cuoteo» que se trató de imprimir a este tipo de iniciativas desde el nivel central, el equipo regional logró una alianza estratégica con la mayoría de los municipios, expresada en una oferta anual que se le hacía a cada uno de ellos para acceder a estos programas. En otras palabras, desde la Coordinación Regional de los Programas Casas y Oficinas de Juventud se enviaba, en octubre de cada año, una carta a cada alcalde describiendo la naturaleza de los programas y, lo más importante, indicando que las responsabilidades administrativas (local, gastos básicos, honorarios del personal, etc.) debían ser asumidos por cada municipalidad. Por su parte, los recursos aportados por el INJ se destinaban en su totalidad a la ejecución de programas de beneficio directo a las instituciones juveniles de la comuna.

Según la programación del INJ central, el año 1997 se «discontinuarían» estas iniciativas. El INJ de la VIII Región el año 1996 ya había traspasado la totalidad de la dependencia administrativa de estos programas a los municipios. Aquello significó que la dicotomía presente en el programa respecto de quién era el «jefe» de los encargados locales de estos programas se zanjó en forma automática a favor de las municipalidades. El INJ, por medio de su Coordinación Programática, sólo mantenía tuición técnica en la elaboración de las iniciativas locales que requirieran recursos institucionales, las que se evaluaban en lo que se conoció como la Red Regional de Oficinas y Casas de Juventud, compuesta por 18 OMJ y 14 Casas de Juventud, con una cobertura de 24 comunas. 
Esta instancia tuvo un enorme éxito. Se constituyó en Red mucho antes que el INJ central presentara iniciativas al respecto. El coordinador de estos programas era el secretario ejecutivo de la red y, además, el coordinador del Grupo de Trabajo Interministerial de Juventud (GTI), lo que aseguraba una discusión que siempre tuvo presente la situación de las comunas en la focalización de recursos provenientes de otras entidades públicas, constituyendo a cada unidad de juventud comunal en aliados estratégicos de entidades como la SEREMI de Educación, Servicio de Salud, FOSIS, DIGEDER y CORECE. En resumen, los recursos que no podía aportar el INJ, se gestionaban a nivel local por medio de este tipo de alianzas. Por lo tanto, cuando se ofertaba a los municipios estos programas, no sólo se les ofrecía algún recurso institucional y la respectiva asistencia técnica, sino que un programa instalado completamente con la capacidad de, por lo menos, duplicar al primer año de funcionamiento los recursos invertidos por la municipalidad.

\section{LA RED REGIONAL DE UNIDADES MUNICIPALES DE JUVENTUD}

Al ocurrir la crisis del INJ, los programas Casas y Oficinas de Juventud estaban consolidados y legitimados a nivel local, lo que motivó a muchos municipios a asumir la totalidad de los costos de mantención, situación que tampoco fue especialmente traumática ya que desde 1996, el aporte directo del INJ se había reducido drásticamente y, como exponíamos, en nuestra región más bien se instalaba una malla programática con recursos de otras instituciones públicas.

El problema radicó en la validez de la interlocución y gestión de la Red, hasta ese momento a cargo del INJ, lo que representaba un problema bastante complicado por cuanto las Oficinas y Casas de Juventud contaban con escaso personal y los recursos que éstos gestionaban —a pesar de ser siempre significativamente más de los asignados en los presupuestos municipales - apenas daban abasto para sus propias necesidades. Además, por situaciones de carácter administrativo y legales se hacía muy difícil que alguno de los municipios «grandes», con más personal y recursos, asumiera la convocatoria de los demás. Era necesario y urgente, entonces, crear un nuevo centro de gestión para la Red.

En reunión de la Red citada para el efecto, inventamos lo que hoy se conoce como la Comisión de Juventud de la Asociación Chile- 
na de Municipalidades, Capítulo VIII Región (ACHM).

Esta comisión se constituyó a partir de las leyes que rigen a los municipios y que favorecen la asociatividad de éstos. En pocas palabras, se planteó al directorio de esa entidad la necesidad de dar continuidad a lo obrado con las Casas y OMJ, para lo cual debería efectuarse una convocatoria a todos los alcaldes y concejales que desearan conformar una comisión de trabajo en el tema juventud. Afortunadamente, la situación en nuestra región ha sido relativamente propicia para este tipo de iniciativas: por un lado Oficinas y Casas legitimadas y reconocidas por las autoridades locales y la comunidad, una Red en funcionamiento legitimada en su trabajo con otras entidades públicas y, para asegurar el éxito de la propuesta, quien fuera coordinador de estos programas en el INJ regional era, además, concejal de la comuna de Concepción.

En noviembre de 1998, legalmente y en un acto electoral formal al interior de la ACHM, se constituyó la Comisión, cuya primera misión fue constituirse en Secretaría Ejecutiva de la Red, retomar el contacto formal con los municipios a fin de sostener la voluntad de los alcaldes y concejales para el mantenimiento de estos programas y, a la vez, requerir opiniones a fin de potenciarlos a nivel local.

A partir de estas primeras iniciativas, la comisión cambió la denominación de las Oficinas y Casas a las de Unidades Municipales de Juventud (UMJ), reconociendo como Unidades no sólo a las antiguas depositarias de los programas del INJ sino que, además, incorporando a la Red a aquellos municipios que, por falta de recursos, no tienen la posibilidad cierta de invertir en personal o infraestructura particulares. Nuestra Red de UMJ considera, entonces, la figura de los Sectorialistas Locales de Juventud, muchas veces un funcionario de DIDECO o SECPLAC que, si bien asume otra serie de funciones, nos brinda la oportunidad de una interlocución personalizada para las iniciativas que desplegamos.

Nuestras región cuenta, hoy, con 30 municipios adscritos a la Red, con una cobertura ideal del 84\% de la población juvenil regional.

\section{EXPERIENCIAS E INICIATIVAS}

La fortaleza de esta Red se reflejó en el enorme éxito que tuvimos al proponer al Gobierno Regional del Bío Bío (GORE) la creación de un Fondo Concursable, con recursos del FNDR, destinado a las organizaciones juveniles a partir una iniciativa que conocimos y que 
sólo contemplaba un concurso para las áreas de adulto mayor y cultura.

Básicamente, se trató de sensibilizar a los consejeros regionales sobre la necesidad de invertir directamente en la gente. En este caso, la gente joven y sus institucionalidades. La Red propuso, además de la creación del Fondo de Juventud, una metodología de trabajo, tipología de proyectos, formatos para la convocatoria y pautas de evaluación, que fueron aceptadas en casi su totalidad no sólo para la temática juvenil, sino que para las otras dos áreas que interesaban al Consejo Regional.

Además, logramos convencer a los consejeros regionales sobre un aspecto de primordial importancia para el éxito del proceso en general: que los recursos fueran postulables sólo por organizaciones juveniles a través de los municipios. Es decir, asegurar la inversión directa en las personas y sus instituciones, en proyectos concretos y de interés inmediato para los individuos y sus comunidades, evitando que postularan otras instituciones públicas y las universidades como contempla el reglamento de postulación a los fondos del FNDR.

Ya en enero del 2000, la iniciativa ha cristalizado en una convocatoria a concurso de proyectos para el año 1999 y 2000, por trescientos millones de pesos, con más de 180 proyectos presentados para el año 99 y la distribución total de los recursos contemplados para ese año en 64 proyectos aprobados por un total de $\$ 100.371 .844$.

Así mismo, la Comisión obtuvo la aprobación de un proyecto presentado a la directiva nacional de la ACHM, con el fin de financiar un pequeño equipo de trabajo, apoyo y seguimiento a los municipios que ganaron proyectos juveniles (39 de 52 existentes en la región). Además, al ser una Comisión constituida de acuerdo a la Ley Municipal, la ACHM, Capítulo VIII Región, ha facilitado oficinas y dependencias en el local que ésta posee, además de todo el soporte administrativo de la misma.

\section{LOS DESAFÍOS VENIDEROS}

A raíz de los contactos desarrollados con FOSIS, INJUV, SEREMI de Gobierno y Consejo Regional, en las mesas en que se discutió y aprobó definitivamente el fondo concursable en el área de juventud, la Comisión y la Red han asumido dos líneas de acción para el período 2000. 


\section{a) Una política regional de juventud}

Como hemos expuesto, en la VIII Región del Bío Bío se ha mantenido y fortalecido una institucionalidad juvenil en el ámbito público a partir del compromiso que diversos actores, en especial los municipios, han asumido desde hace bastante tiempo en esta temática.

Precisamente, desde el mundo municipal ha surgido con fuerza la idea de no esperar más una respuesta, que el Estado parece no ser capaz de dar, sino que, en provecho de nuestras propias experiencias, avanzar en el diseño de nuestra respuesta sobre el particular.

Por lo tanto, estamos muy avanzados en la creación de una Mesa Regional de Juventud que reúna en un trabajo metódico a los representantes regionales de ministerios, servicios y reparticiones públicas, así como al mundo privado y la institucionalidad juvenil existente en la región.

Sobre el particular, ya se estableció una alianza estratégica con el Departamento Comunal de Jóvenes de Concepción, el FOSIS, la SEREMI de Gobierno y la Comisión de Desarrollo Social y Cultural del Consejo Regional, así como el INJUV nacional, destacando un proyecto que la Comisión, en su calidad de Secretaría Ejecutiva de la Red de UMJ, envió en agosto del 99 a la División de Organizaciones Sociales del Ministerio Secretaría General de Gobierno, con la finalidad de financiar algunas de las actividades que este desafío representa. Este proyecto ha sido aprobado y sólo falta concretar el cronograma del presente año con la contraparte regional.

El producto esperado no es otro que contar, por primera vez en la región y el país, con una propuesta clara, formal, práctica y ejecutable, sobre el tema juvenil para ser entregada a las nuevas autoridades regionales y nacionales, sean éstas públicas o del mundo privado, con la finalidad de ser incorporada a las estrategias de desarrollo pertinentes.

\section{b) Fortalecimiento institucional}

El modelo de institucionalidad juvenil desarrollado y gestionado por la Comisión y la forma en que ha enfrentado los procesos descritos, presenta una gran debilidad: necesariamente descansa en la voluntad y compromiso de quienes la conforman, es decir, alcaldes y concejales que deben atender sus propias realidades. Además, alguno de ellos deben movilizarse desde comunas lejanas hacia Concepción 
para reuniones y gestiones propias de los compromisos que han asumido al formar parte de la Comisión.

Así mismo los responsables de las Unidades Municipales de Juventud presentan las dificultades propias de los funcionarios municipales para trasladarse periódicamente hasta nuestra cabecera regional, amén de lo deseable que resulta validar y fortalecer la gestión asociativa de los municipios, en especial en el tema que nos interesa.

En atención a lo anterior, hemos desarrollado dos respuestas prácticas al problema:

- $\quad$ Rediseñar la distribución territorial de la Comisión y la Red, descentralizándolas provincialmente, creando Secretarías Ejecutivas Provinciales, adscritas idealmente a las Gobernaciones en el apoyo logístico, a cargo de los concejales y alcaldes miembros de la Comisión que vivan en esas provincias.

Además, realizaremos un llamado específico para que se incorporen más concejales a cada una de las Comisiones Provinciales.

- Concretar la propuesta realizada a la ACHM referida a consolidar un equipo de apoyo a la gestión.

Si bien hemos avanzado en la creación de un pequeño equipo de apoyo a los municipios según lo descrito anteriormente, este año avanzaremos en incorporar herramientas como convenios para prácticas profesionales en la ACHM, para lo cual hay conversaciones avanzadas con algunos institutos profesionales y universidades.

Así mismo, concretaremos la propuesta relacionada con que los municipios con más recursos y con equipos profesionales más desarrollados en el tema juventud, faciliten algunos de sus funcionarios a la Comisión, ya sea por algunas horas a la semana o para el desarrollo de proyectos específicos que beneficien a la Red. La idea, en definitiva, es que los recursos humanos altamente calificados que poseen las UMJ puedan entregar sus aportes más allá de los límites de sus comunas. Para lo anterior, hemos propuesto a la ACHM que aquellos funcionarios cooperadores sean priorizados en el acceso a becas de perfeccionamiento profesional y otras instancias similares. 